\title{
On-Orbit Servicing Missions: Challenges and Solutions for Spacecraft Operations
}

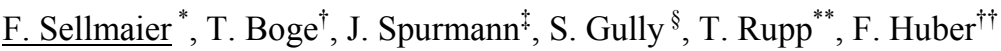 \\ German Space Operations Center (GSOC) \\ DLR Oberpfaffenhofen, D-82230 Wessling, Germany
}

\begin{abstract}
[Abstract] The DLR German Space Operations Center (GSOC) is currently involved in the preparation of two On-Orbit Servicing missions, DEOS and OLEV. Due to the many new challenges within those missions the ground segment design requires new concepts. Accordingly, the paper presents the challenges and solutions regarding the communication architecture including teleoperation and extended contact time. Additionally, we discuss a method of vision based navigation which bridges the gap between absolute and purely geometric navigation. Finally, an integrated system test including GSOC's new EPOS facility is described.
\end{abstract}

\section{Nomenclature}

$\begin{array}{ll}D E O S & =\text { DEutsche Orbitale Servicing Mission } \\ E P O S & =\text { European Proximity Operation Simulator } \\ O L E V & =\text { Orbital Life Extension Vehicle } \\ O O S & =\text { On-Orbit Servicing } \\ R v D & =\text { Rendezvous \& Docking }\end{array}$

\section{Introduction}

$\mathrm{D}$ R's history of On-Orbit Servicing (OOS) goes back to 1987 when TV-Sat 1 was launched. Unfortunately, the failure of one solar panel to deploy curtailed operations severely since the payload could not be activated. After several attempts to repair the spacecraft from ground, it was finally placed in a graveyard orbit in 1989. This incident triggered the idea of a rescue satellite with TV-Sat 1 as an early candidate for OOS studies. SETTELMEYER ${ }^{1}$ et al. (1998) presented the concept of an Experimental Servicing Satellite (ESS) including the design of a capturing tool for the apogee engines of geostationary satellites (patented by DLR). Further concept studies such as TECSAS and CX-OLEV followed. As a result of this research and follow up technology projects like the ROKVISS ${ }^{2}$ experiment on the ISS or the ETS VII contribution, DLR has extensive experience in space robotics (see LANDZETTEL $^{3}$ et al. (2006) and references within) as well as in the field of close formation flying and proximity operations (Tandem-X, PRISMA ${ }^{4}$, EPOS).

Meanwhile, OOS has become part of the space programs of the US, Japan, Canada and Germany. A milestone was set with the successful completion of DARPA's Orbital Express ${ }^{5}$ (OE) mission in 2007. The goal of OE was to demonstrate the ability to autonomously perform Rendezvous \& Docking (RvD) operations including maintenance activities like refueling. In contrast to the goals of OE, the focus of DLR is to capture non-supportive and/or not specially prepared client spacecraft. By "non-supportive" we mean that there is no support with respect to attitude and orbit control of the client, e.g. when the client is non-operational. "Not specially prepared" means that the client satellite does not have a special docking port or retro reflectors used for vision based navigation. This is pursued with DLR's involvement in the two OOS projects DEOS and OLEV. The goals of DEOS are to demonstrate the capture of a tumbling and non-supportive client satellite in low earth orbit and a controlled de-orbiting of the mated

\footnotetext{
*Business Development, DLR Space Operations, florian.sellmaier@dlr.de

${ }^{\dagger}$ Spaceflight Technology, DLR Space Operations, toralf.boge@dlr.de

* Spaceflight Technology, DLR Space Operations, joern.spurmann@dlr.de

$\S$ Ground Data System, DLR Space Operations, sylvain.gully@dlr.de

${ }^{* *}$ Spaceflight Technology, DLR Space Operations, thomas.rupp@dlr.de

${ }^{\dagger}$ Director, DLR Space Operations, felix.huber@dlr.de
} 
system. OLEV is a commercial project with the goal to extend the lifetime of geostationary communication satellites whose fuel has been depleted. A brief description of DEOS and OLEV is given in section II.

The OOS mission DEOS and OLEV pose several challenges to spacecraft operations:

1) Communication: Capturing a tumbling client as well as RvD operations set requirements far beyond the capabilities of standard communication architecture: Delay Time and Jitter of the signal have to be minimized; shading and interference have to be avoided.

2) Navigation: A gap between absolute navigation (e.g. ranging) and purely geometrical relative navigation (e.g. stereo camera) has to be bridged by an alternative method.

3) Verification: Approach navigation, capture and docking algorithms should be thoroughly tested on ground first. A sophisticated test facility including gravity compensation and contact dynamics is necessary.

Concepts and solutions to the above listed challenges will be discussed later in sections III, IV and V. Aspects regarding the Flight Operation System (FOS) of DEOS and OLEV are discussed in EBERLE ${ }^{6}$ et al. (2010).

\section{Present OOS projects at GSOC}

\section{A. Technology Missions DEOS}

The primary goals of the technology demonstrator DEOS are (1) to capture a tumbling non-supportive client satellite with a servicer spacecraft and (2) to de-orbit the coupled configuration within a pre-defined orbit corridor at end of mission. Secondary goals are to perform several Rendezvous, Capture and Docking scenarios as well as orbit maneuvers with the mated configuration. Therefore the Servicer is equipped with an active Attitude and Orbit Control System (AOCS) and both a manipulator and a docking port (see Figure 1).

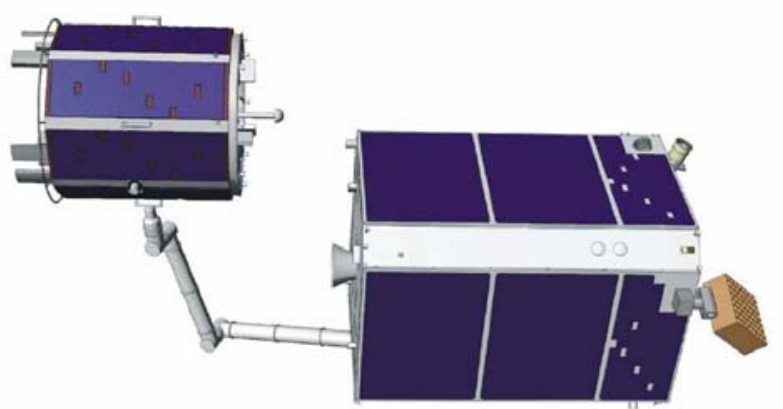

Figure 1: DEOS Client (left) and Servicer (courtesy STI)

Since the initial experiment conditions like tumbling rate of the client have to be set several times, the client is provided with an active Attitude Control System (ACS). For DEOS the expression "non-supportive client" has to be understood in a sense that the clients ACS must not be used during the capture maneuver described above. Additionally, both Servicer and Client are equipped with GPS / RGPS receivers. Again, GPS and RGPS are not used for nominal approach navigation and capture but as a reference for subsequent evaluation or in case of collision avoidance. The sensor system used for the nominal approach navigation is a vision based system using mono and stereo cameras.

Similar to Orbital Express the mission philosophy is to subsequently "crawl, walk and run": Both spacecraft, client and servicer, will be injected together in an initial low earth orbit (LEO). Starting with the mated configuration the complexity of the experiments is stepwise increased over mission period. One of the challenges operating DEOS is the continuity of a communication link from ground to LEO. Therefore the DEOS Servicer will be equipped with a Ka-Band link to Geo-Relay satellite as an option to direct space to ground communication (see section III). The DEOS project is presently entering a phase B study financed by the German Space Agency.

\section{B. Commercial Mission OLEV}

OLEV is a purely commercial project managed by a European consortium including a strong DLR participation. The business case of OLEV is to build an orbital "tug boat" which is able to dock on high value, geostationary communication satellites and to take over Attitude and Orbit Control in order to extend the clients lifetime after its fuel has been depleted (Figure 2). Beside life extension OLEV can be used for fleet management purposes like relocation to other geostationary positions or disposal to graveyard orbit.

The core element of OLEV is the capturing tool (patented by DLR) which enables OLEV to dock on

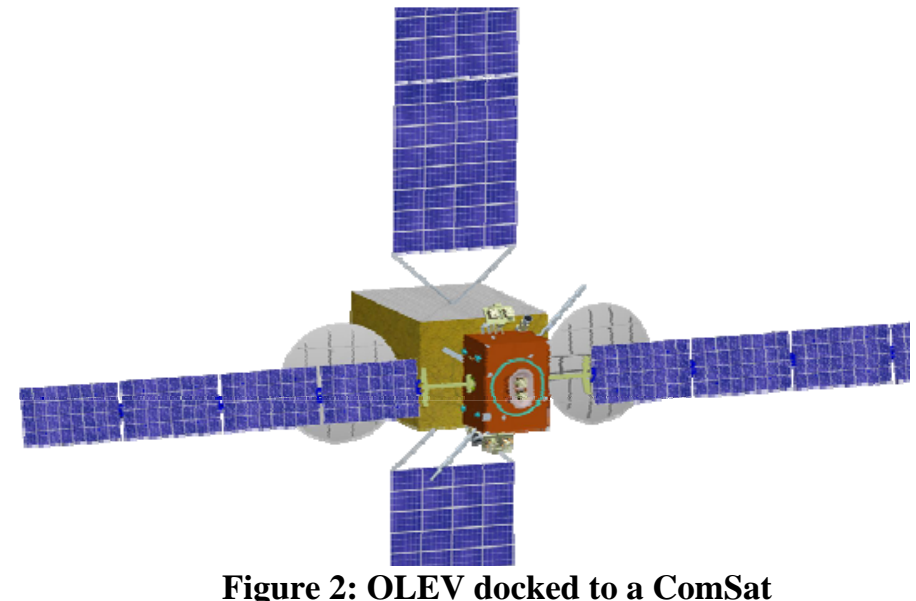

Figure 2: OLEV docked to a ComSat 
the apogee engine of the majority of the existing geostationary communication satellites. The capturing tool is designed to allow OLEV to dock / undock several times. The OLEV platform is equipped with six "Hall Effect Thrusters" (HET): Two of them are used for the transfer from the Geostationary Transfer Orbit (GTO) to the Geostationary Orbit (GEO), the other four $(2 \times 2)$ are dedicated for the station keeping. The electric propulsion system enables OLEV to perform station keeping of the mated configuration for approx. twelve years depending on the client mass.

There are several challenges regarding the operation of OLEV: One of them is that the mated configuration's center of mass (COM) lies outside OLEV's body dimensions. Since the thrust vector for station keeping needs to point through the COM the N/S station keeping maneuvers will always result in a radial component which has to be compensated after 12 hours. This yields to a more complex maneuver strategy and an enhanced fuel consumption of a little more than $100 \mathrm{~m} / \mathrm{s}$ per year instead of approx. $53 \mathrm{~m} / \mathrm{s}$ per year. Other challenges will be described in the subsequent sections. The navigation concept of OLEV is to use ranging for absolute navigation and to hand over to relative navigation at the distance of a few $\mathrm{km}$ (see section IV). For relative navigation a set of six rendezvous cameras (far, mid and close range, redundant) is used.

The OLEV project has finished a delta phase B study; the present focus lies on financial engineering.

\section{Communication}

Both RvD maneuvers and the capture of a client satellite require either a new quality of communication links or a very high degree of autonomy. Hence the requirements on the communication link are defined by risk analysis and a balance between the costs for autonomy versus cost for an improved communication concept.

\section{A. Continuity of the communication link}

In a typical low earth orbit there is maximum of approx. 8 minutes contact time per path if only one ground station is used. Since the DEOS servicer requires a minimum of 20 minutes contact time while capturing the tumbling client a different solution has to be found. One solution is to combine a chain of ground stations as demonstrated in Figure 3 which provides a continuous telemetry (down link) data stream for more than 20 minutes. However, telecommand (uplink) will be interrupted since a handover from one ground station to another requires approx. one minute. Since the earth is rotation the depicted chain of ground stations can be used every 12 hours only. Another more elegant solution to extend contact time is to use a KaBand intersatellite link to a geo-relay satellite between servicer and a dedicated ground station. However, the availability of geo-relay satellites and/or costs may be a limiting factor to this strategy.

The situation is far simpler for the geo-stationary mission OLEV for which a continuous communication link is available in principle. However, since the final approach towards the apogee engine of the client occurs along R-bar, shading and interference may disrupt the communication link to the servicer. A solution to this problem is a world wide network of ground stations during the R-bar approach. A simple simulation (Figure 4) demonstrates that there is always at least one ground station (red dots) visible beside the outline of the client satellite (blue cross). Accordingly, there is at least one ground station visible for each of the two servicer antennas. Coming closer to docking $(20 \mathrm{~m}-0 \mathrm{~m})$ the visibility will increase again since the S-band antennas are located on beams (Figure 2). Possible interference by the client has to be investigated in an RF compatibility test.

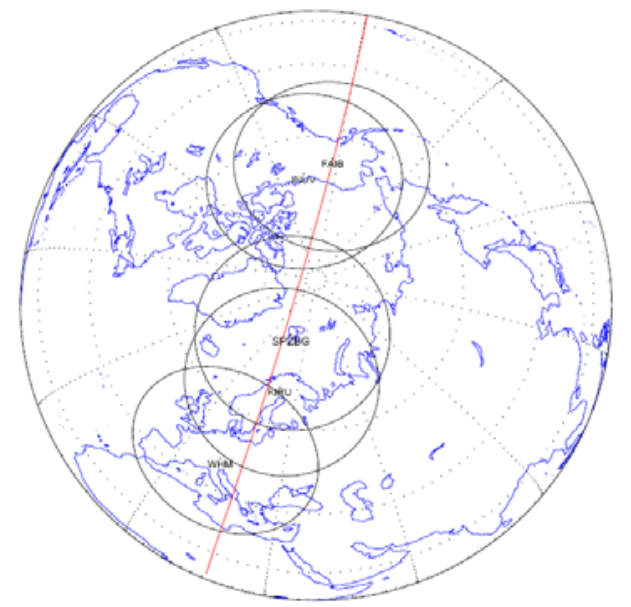

Figure 3: Chain of ground stations to extend contact times in low earth orbit (DEOS)

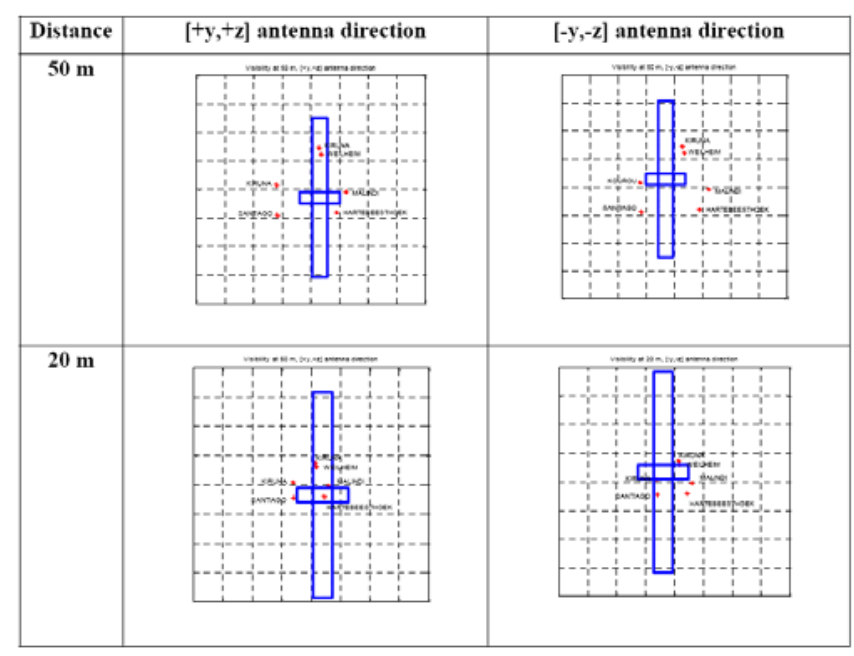

Figure 4: Visibility of ground stations during R-bar approach in geostationary orbit (OLEV) 


\section{B. Teleoperations: Delay Time and Jitter}

The requirement to minimize delay time and jitter is driven by the robotic operations in the final Rendezvous and Docking / Capture phase. The payload control system (PCS) of DEOS requires a delay time of less than $500 \mathrm{msec}$ (round trip) during the robotic phase, i.e. the capture of the tumbling client. For OLEV the requirements are less stringent since the approach velocities in GEO are much smaller and, additionally, the client is not tumbling but 3axis stabilized. However, a delay time smaller than one second (round trip) is recommended. The problem is that the standard communication architecture introduces a delay time of typically $2-5 \mathrm{sec}$, mainly due to electronic components on ground. Additionally, automatic switching of redundant lines may cause unpredictable jitter.

A solution to both problems is to connect the PCS directly with the cortex (CTX) of the teleoperation antenna with a dedicated non-redundant high rate TM/TC link (Figure 5: blue lines). The 34 Mbps line introduces a very small delay time of $2,5 \mathrm{msec}$ round trip. This solution is used for ROKVISS ${ }^{3}$ operation since several years. Hence, the over all delay time can be reduced to smaller than 500 msec round trip (including image processing). The line is not automatically redundant. This avoids jumps in the delay time which limits the jitter. In case of a component failure the communication link can be switched back manually to the standard multi mission TM/TC link (green lines). Since teleoperation is needed during RvD maneuvers only, manual redundancy is not a serious problem.

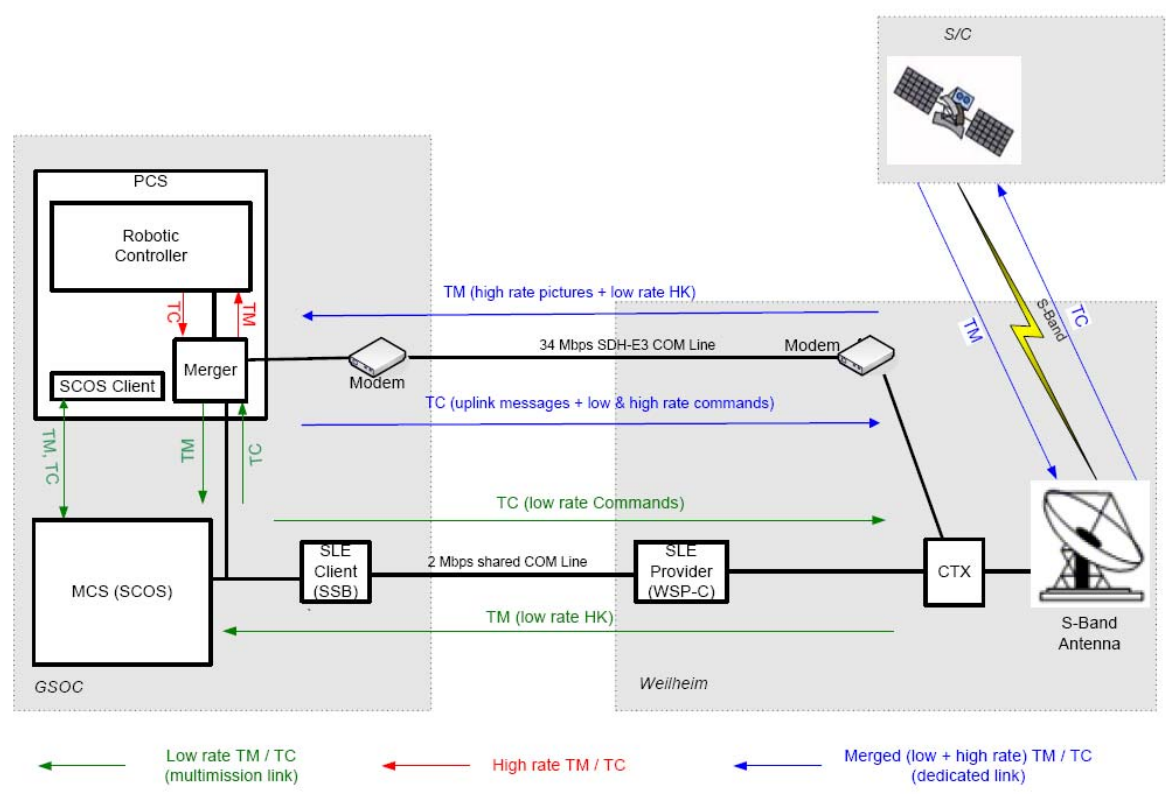

Figure 5: Communication architecture to minimize delay time and jitter

\section{Navigation}

The major design driver for the navigation system of a spacecraft performing RvD operations is the duration of autonomous operation. For the presented LEO mission the requirement is to achieve one orbit of autonomous operation. Due to orbital perturbations this requirement yields a measurement accuracy of one percent of the range between target and servicer for the navigation sensors ${ }^{7}$, the socalled " $1 \%$ rule". However, for missions in GEO this requirement can be slightly relaxed as there is a permanent communication link and the orbital period is much longer. Hence, adjustments can be performed much quicker. Having identified the design driver navigation sensors can now be selected for each mission phase (Figure 6).

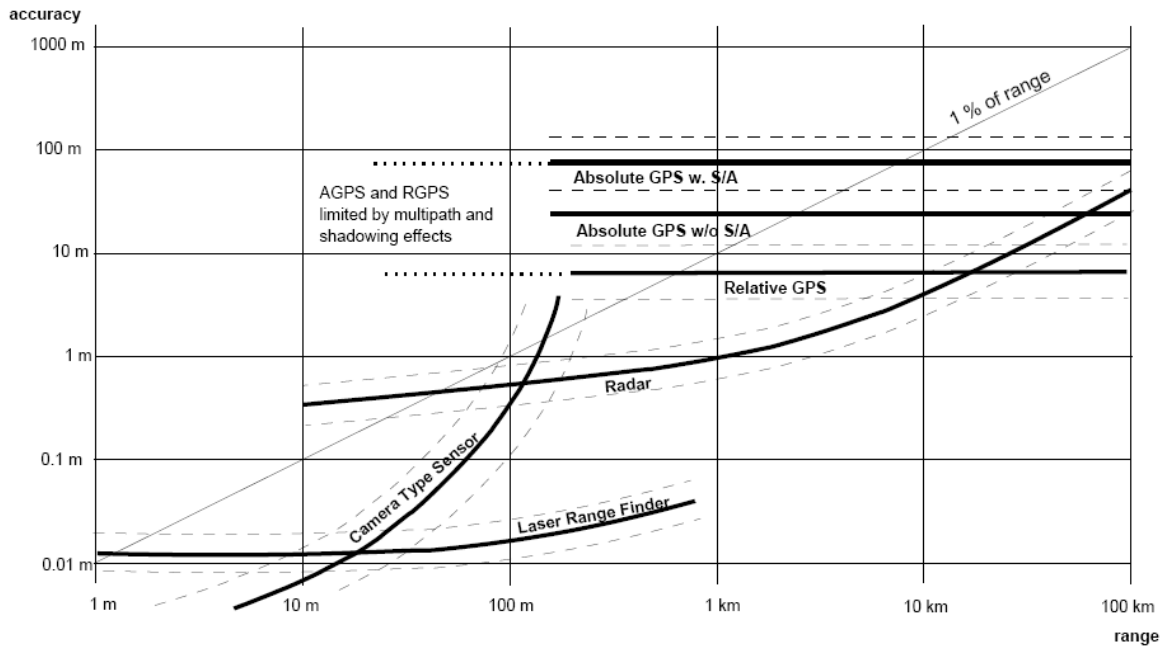

Figure 6: Typical operational ranges and measurement accuracies of rendezvous sensors (from Fehse ${ }^{7}$ ). The diagonal indicates the "1\% rule". 


\section{A. Absolute Navigation}

During phasing, in which the phase angle between target and servicer is reduced and finally an entry gate for the far range rendezvous is achieved, absolute navigation can be performed with ground in the loop. Different solutions are available, whereby Ranging or GPS are most commonly used. While both techniques are applicable in LEO, GPS is much more likely to be used at this altitude as the achievable measurement accuracy is smaller than ten meters (slight technology advancement compared to Figure 6). Accordingly, the servicer in LEO will be equipped with a GPS receiver. The target however, might be inactive or not equipped with an active GPS receiver. In this case radar tracking from ground is the best solution, allowing similar accuracies below ten meters ${ }^{8}$. Due to the " $1 \%$ rule" described above the absolute navigation in LEO can be used up to a relative distance of $1 \mathrm{~km}$.

In GEO GPS navigation data is not available in an operational sense and ranging is the selected technique. The accuracy lies around $100 \mathrm{~m}$ in along track and several hundred meters in cross track direction. As a result a relative distance of several kilometers has to be sustained, due to a relaxed " $1 \%$ rule" for the rendezvous sensors (Figure 6).

\section{B. Relative Navigation Options}

Having identified the minimum relative distance between target and client satellite in the kilometer range for absolute orbit determination, the necessity of relative navigation sensors is obvious. By placing the measurement equipment onboard the spacecraft, it is no longer positioned on ground where a very precise knowledge of the reference location would be available. Instead it is located in space where it is exposed to the orbital dynamics and the harsh space environment. Accordingly the measurement biases will increase.

The relative navigation sensors considered are Relative GPS (RGPS), Radar, LIDAR, or Camera Type Sensors. RGPS would yield an accuracy of several meters, reducing the relative distance to several hundred meters (Figure 6). However, a dedicated receiver needs to be present on the target satellite for relative GPS and additionally an intersatellite link needs to be established. As the mentioned OOS missions are intended to dock with non-supportive spacecraft not equipped with receivers or link functionality a different approach needs to be investigated.

Radar or LIDAR systems would be appropriate to lower the achievable relative range to orders of ten meters (Figure 6). On the other hand both systems would also pose difficult constraints on the mass and power budget of the space segment. As both budgets are limited in the mentioned missions those system do not yield a perfect solution either.

\section{Camera Based Relative Navigation}

As a result a camera type navigation system will be used for relative navigation. Such a system will already be used in the final approach phase for pure geometrical navigation for two reasons: it provides the best accuracy and a monitoring of the capturing process is possible. Position and pose estimation using camera type navigation is, however, very restricted with respect to relative range. Usually features on the target spacecraft are identified on the camera focal plane to estimate target's position and orientation. However, the estimation works only as long as the features are clearly detectable and distinguishable on the camera focal plane. Preliminary picture generation shows however that this is only possible within a distance of several tens of meters between target and servicer (Figure 7), which is also verified by the analysis of FEHSE $^{7}$ (Figure 6). The pictures are generated for a CCD size of 512 × 512 pixels and a pixel size of $25 \mu \mathrm{m}$ for both cameras, while the far range camera (FRC) has a focal length of $200 \mathrm{~mm}$ and the mid range camera (MRC) of $14 \mathrm{~mm}$. This results in a field of view (FOV) of $3.6^{\circ}$ for the FRC and of $42.4^{\circ}$ for the MRC respectively. The apparent target size results from a spacecraft size of $7 \times 2.5 \mathrm{~m}$ and a solar array wingspan of $28 \mathrm{~m}$. It has to be noted that camera based measurements depend not only on FOV and pixel resolution, but also on other mission parameters like lighting conditions, reflectivity of target's surface, size and shape of the target, the number and size of target features and of course the image processing capabilities (see also MIRAVET ${ }^{13}$ et al., 2008) Hence the position and pose estimation quality is restricted by

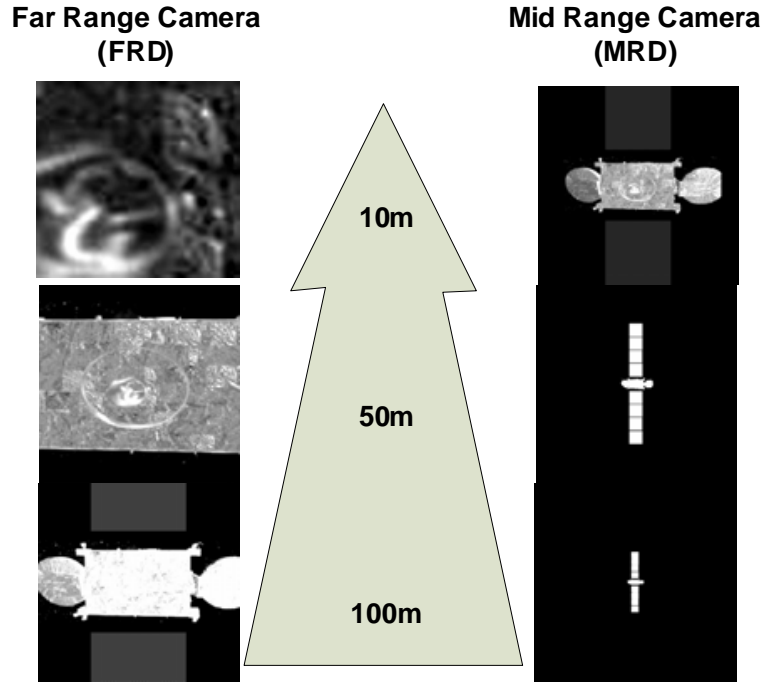

Figure 7: Simulated images for Far and Mid Range Camera at different relative distances 
those characteristics, which has to be analyzed more in-depth.

If the target features are no longer detectable on the camera focal plane the relative range might however be calculated as suggested by WOFFINDEN ${ }^{9}$ if the target spacecraft size is known. By dividing the known spacecraft size by the apparent diameter on the camera focal plane the range can be obtained. However, the computation is only possible as long as the targets apparent diameter on the camera focal plane is larger than one pixel: even if a far range camera with a FOV of $3.6^{\circ}$ is used (Figure 7), such a measurement is only possible up to a relative range of several hundred meters.

A similar argument also holds for the use of stereo cameras since the baseline i.e. the distance between both cameras is even less than the size of the client which is the baseline used in the method described previously. The only way to improve a stereoscopic method is the use of an optical bench which is not appropriate with respect to the servicer's mass budget.

The conclusion from the above is that a transition zone remains between several hundred meters to several kilometers, for which a determination of the relative range is not sufficiently accurate. Hence, without using Radar or LIDAR rendezvous sensors the remaining gap starting at the handover point from absolute to relative navigation has also to be covered by an improved camera based navigation system.

\section{Angles-Only Navigation}

As a solution to this problem a method called angles-only navigation will be implemented to acquire the relative range within the mentioned transition zone. The method is well know and widely applied in naval applications, orbit determination, target tracking, lunar and interplanetary optical navigation and homing missile applications ${ }^{9}$.

The relative trajectory between target and servicer can be defined by the relative distance $r$ and the line-of-sight (LOS) angles azimuth $\alpha$ and elevation e. The basic principle of the angles-only navigation is then to measure the LOS angles with the according time very accurately (Figure 8), as soon as the target can be detected as a moving star in front of the background star field by the FRC. The obtained measurements are then used to update a Kalman Filter, which propagates the orbit onboard the servicer or on ground. Apart from an initial guess of the relative range the Kalman Filter uses the final spacecraft states from absolute navigation, the orientations of the spacecraft, biases (gyro bias or camera misalignment) and noise terms to determine the state vector of the target spacecraft ${ }^{9}$. By iterative propagation and a continuous update process of the filter, the accuracy of the measurements and most important that of the relative range will improve.

The major problem of angles-only navigation according to WOFFINDEN ${ }^{10}$ (2009) is however, the inherent limitation in determining the relative range with adequate accuracy. If the relative motion between servicer and target does not change (e.g. Vbar hold point), and equivalently the continuous LOS measurement profile is not altered, a precise

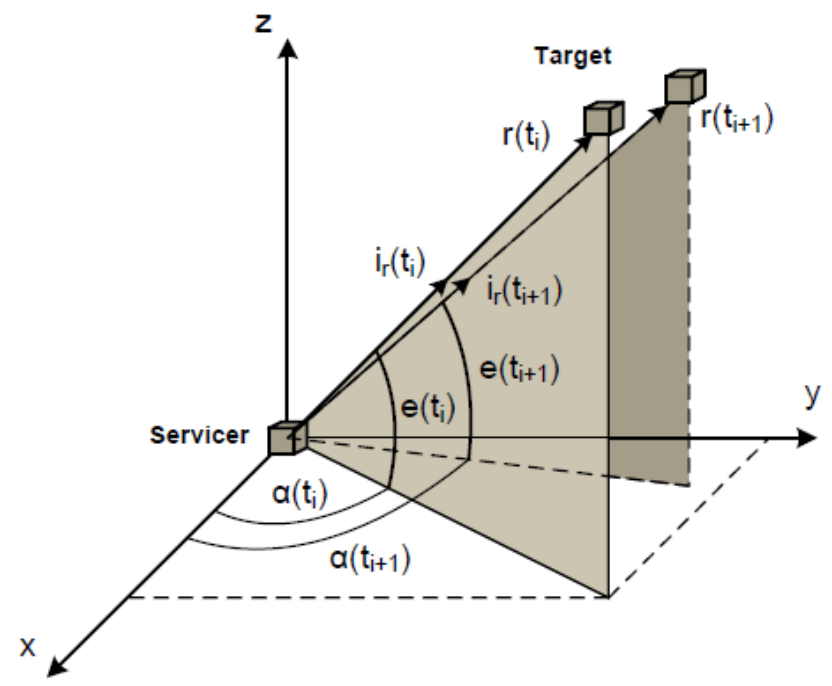

Figure 8: Geometry of angles-only measurements determination of the relative range is not possible. According to his research only the family of relative orbits ${ }^{10}$ can be determined in this case. Nevertheless, application of arbitrary maneuvers forces a change of the relative position and the relative range can be determined accordingly. As a result from this finding specific trajectory profiles have to be planned at the handover point from absolute to relative navigation. Thus, approach of the target by impulsive maneuvers in tangential or radial direction is advantageous, as the resulting hopping trajectories or fly around maneuvers (Figure 9) allow for a proper anglesonly navigation $\left(\mathrm{ESA}^{11} 1998\right.$ and FEHSE $\left.{ }^{12} 2009\right)$. 
Additional accuracy improvements can further be achieved upon different Kalman filter implementations. Literature ${ }^{14}$ shows that the Unscented Kalman Filter (UKF) shows slight superiority over the Extended Kalman Filter (EKF).

However, our interest is the direct estimation of relative orbit elements (ROE) from the angles only measurement by a Kalman Filter. Although SCHMIDT and LOVELL already provided an estimation of ROEs from LOS measurements ${ }^{15}$ a different set of ROEs as proposed by D'AMICO and MONTENBRUCK $^{16}$ will be used instead. Those are more geometric in nature compared to the state vector consisting of position and velocity, although the level of abstraction is rather high. Anyhow, orbital control and perturbation modelling is much easier to handle with a state vector comprised of ROEs $^{16}$, both of which are highly important for rendezvous operations.

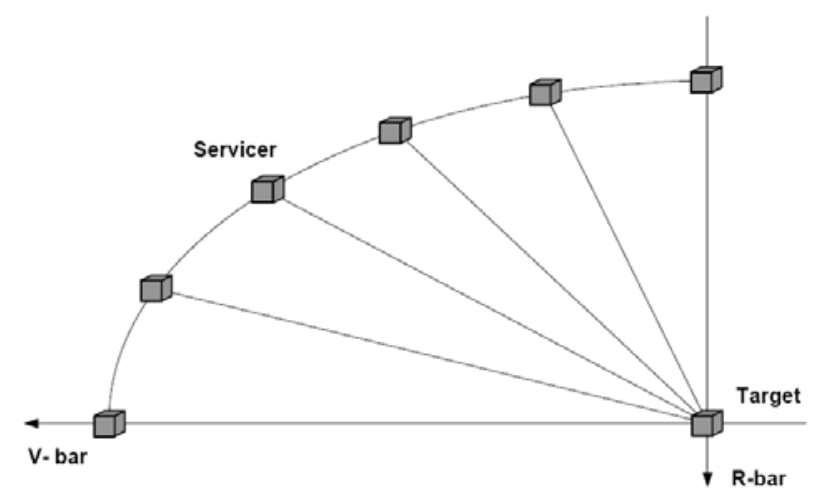

Figure 9: Angles-only measurements during fly around maneuver

\section{Verification}

The requirements of OOS missions on guidance, navigation and control (GNC) are quite different compared to standard spacecraft operations where for instance a communication satellite has to be positioned within a box of $70 \mathrm{~km}$ edge length. The critical phase of OOS missions, the Rendezvous and Docking (RvD) of two spacecraft is a very complex maneuver which requires (relative) position accuracy of a few $\mathrm{mm}$. Additionally, this is connected with difficult communication conditions in low earth orbit (see section III), or with a high risk in case of failure in the (near) geostationary orbit. In consideration of these circumstances an RvD maneuver shouldn't be performed in space for the first time. All RvD maneuvers have to be analyzed, simulated and verified on ground in detail. Classical approaches, e.g. numerical simulations deliver only limited results. Therefore tests or test facilities have to be defined where the entire RvD process including the flight HW of GNC components and systems can be simulated and tested under utmost realistic conditions of the space environment.

\section{A. Requirements on a test facility for OOS missions}

The requirements on testing the missions OLEV and DEOS can be summarized in following three categories.

1) Approach: A test facility should be appropriate to verify sensors and systems within the entire range of vision based relative navigation, i.e. from several $\mathrm{km}$ down to contact (see section IV). For camera based sensors this can be realized in a combination of scaled models and a sufficient range of the test facility. Additionally, the facility shall provide utmost realistic environmental conditions, i.e. the simulation of the sun illumination effect under all angles of incidence and the simulation of the reduced gravity force in orbit.

2) Capture: In order to verify the final "robotic phase" of the RvD maneuver, i.e. the capture of the client satellite, contact dynamics has to be included. This implies a sensor to measure the contact forces and torques and a dynamic model of both satellites (client and servicer) to simulate the reaction on the contact during the capture process. Furthermore, the requirement to verify spacecraft position accuracy in the range of $\mathrm{mm}$ the test bed has to guarantee accuracy in the sub-millimeter range.

3) Integration: The facility shall be able to support an integrated system test including RvD system hardwarein-the-loop. It should further be connected to the control center infrastructure including the mission control system (MCS) and the payload control system (PCS) as well as a realistic ground data infrastructure with respect to delay time and jitter. Finally, the facility shall be used for operator training and mission support.

\section{B. DLR's experience with RvD simulation}

DLR has more than two decades experience in the field of simulating RvD processes. The former EPOS facility (European Proximity Operations Simulator) was a test bed jointly developed by ESA and DLR. It was designed to simulate rendezvous maneuvers of spacecraft over the last 12 meters of the rendezvous phase (without docking). The main utilization was the test and verification of the ATV RvD sensors and systems. 
However, although extensive experience was gained with the original EPOS facility, the OOS mission requirements for accuracy and dynamics exceed the specification of the former facility. Hence, DLR decided to build up a completely new simulation facility.

\section{Characteristics of DLR's new EPOS-Facility}

The new EPOS facility comprises a hardware-in-the-loop simulator based on two industrial robots for physical real-time simulations of rendezvous and docking maneuvers (Figure 10). One of the industrial robots is mounted on a $25 \mathrm{~m}$ rail system to simulate the 6 degree of freedom (DOF) of the first spacecraft, the other industrial robot is mounted at the end of the rail to carry the second spacecraft (6 DOF).

The utilization of standard industrial robotics $\mathrm{H} / \mathrm{W}$ allows a very high flexibility related to different application scenarios. The robots are capable of carrying up to $200 \mathrm{~kg}$ payload. It should be mentioned that both, client and servicer model can be either mounted on robot 1 or 2 (compare Figure 11) - there are pros and cons for both scenarios. All necessary cables for sensors etc. are also available on the rail mounted robot.

To achieve best simulation and verification results the accuracy of the entire facility was extensively evaluated. Additionally, an optical high-accuracy measurement-device will guarantee position accuracy in sub-millimeter level. Furthermore, a lot of effort was made to increase the command frequency to $250 \mathrm{~Hz}$ which is an important precondition to simulate real time contact dynamics.

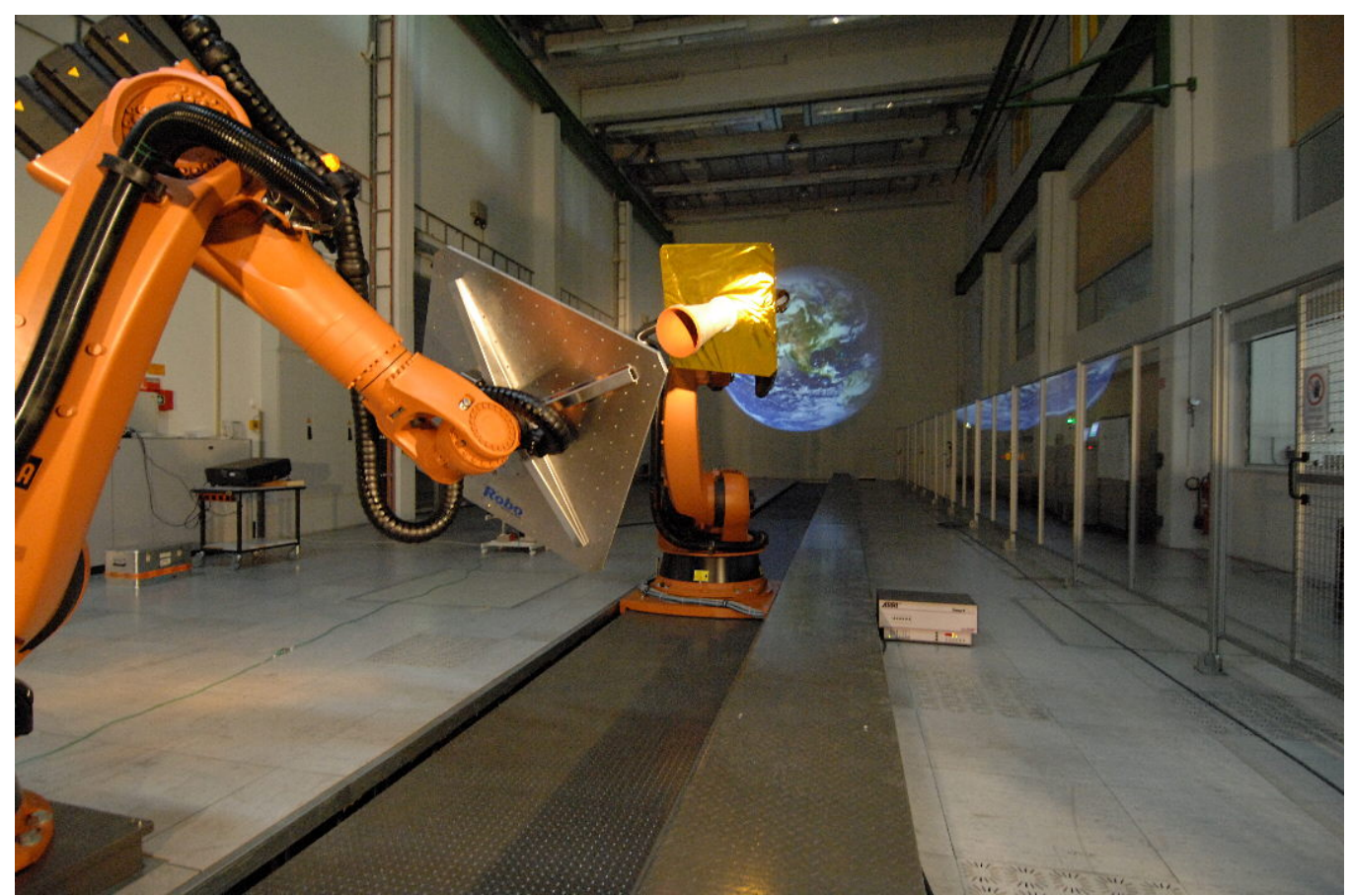

\section{Figure 10: The new EPOS-facility at GSOC}

Besides the robotic hardware, a PC-based monitoring and control system is connected to the test bed which can be divided in three levels (see Figure 11):

- The local robot control where each axis of the robot is separately controlled

- The facility monitoring and control system (FMC) where the entire facility is controlled in real time

- The application control system where the actual RvD-simulation application is running 


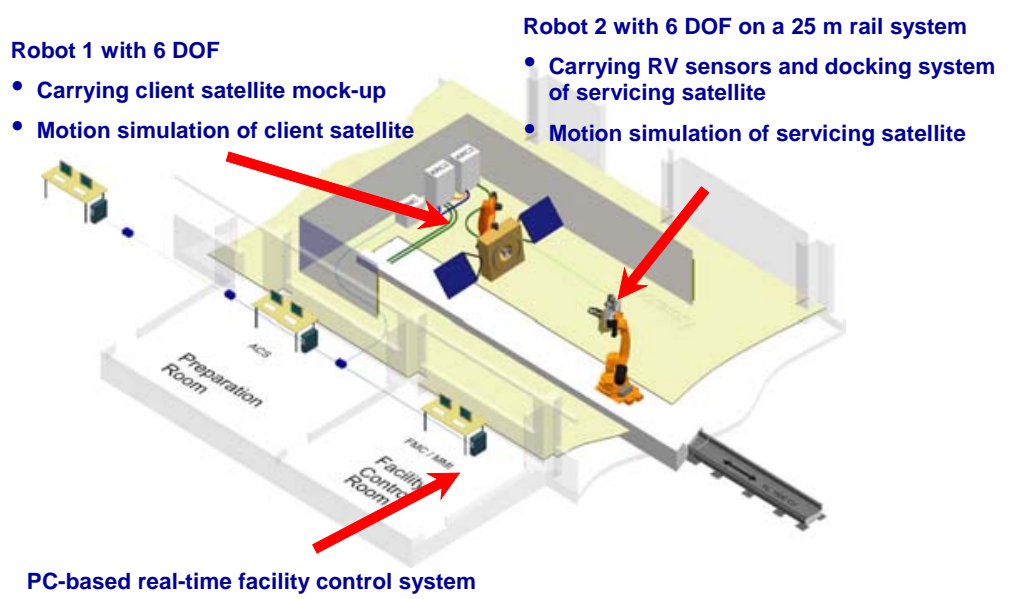

\begin{tabular}{|l|c|}
\hline \multicolumn{1}{|c|}{ Parameter } & Value \\
\hline & \\
\hline Motion Ranges: & up to $25 \mathrm{~m}$ \\
\hline Range: & $>360 \mathrm{deg}$ \\
\hline Line-of-Sight (Azimuth, Elevation): & $360 \mathrm{deg}$ \\
\hline Roll/Pitch/Yaw: & \\
\hline & \\
\hline Accuracies: & $0.5 \mathrm{~mm}$ \\
\hline Range: & $0.01 \mathrm{deg}$ \\
\hline Line-of-Sight (Azimuth, Elevation): & $0.01 \mathrm{deg}$ \\
\hline Roll/Pitch/Yaw: & \\
\hline & $250 \mathrm{~Hz}$ \\
\hline Command Frequency: & \\
\hline & up to $200 \mathrm{~kg}$ \\
\hline Payload: & \\
\hline
\end{tabular}

Figure 11: Facility concept and key parameters of the new EPOS-facility ${ }^{17}$

\section{A typical RvD Hardware in the Loop Scenario}

A typical set-up of the EPOS facility for a DEOS RvD simulation scenario is shown in Figure 12. For such "hardware in the loop" scenario the RvD sensors and the robotic manipulator arm are mounted on one robot and a typical satellite mockup of the client satellite is mounted on the other robot. The RvD sensors can measure the relative position and attitude of the client satellite and the onboard computer calculates on this basis the necessary thruster or reaction wheel commands. Those will feed in a real time simulator. This dynamic simulator computes for the next sample an update of the state vector (position and attitude of the spacecraft) based on all relevant forces and torques resulting from environmental conditions or control

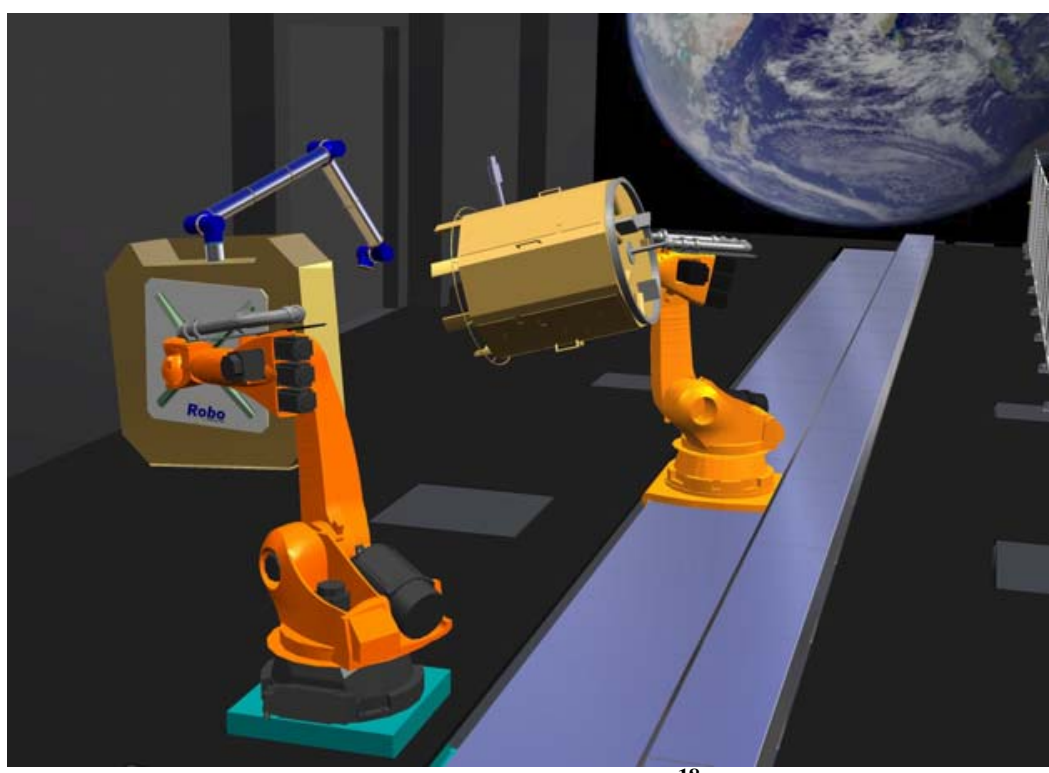

Figure 12: EPOS simulation set up for DEOS ${ }^{18}$ activities. Then the state vector for the new sample will be commanded to the facility.

The possible design of a system integration test for the OLEV scenario is shown in Figure 13. As shown, the scenario integrates the EPOS facility, the satellite Hardware-In-the Loop simulator (OSTF HIL Simulator), the control center infrastructure like Operations Control System (OCS - called MCS in Figure 5), PCS (including Merger) and parts of the communication architecture (e.g. the CORTEX). 


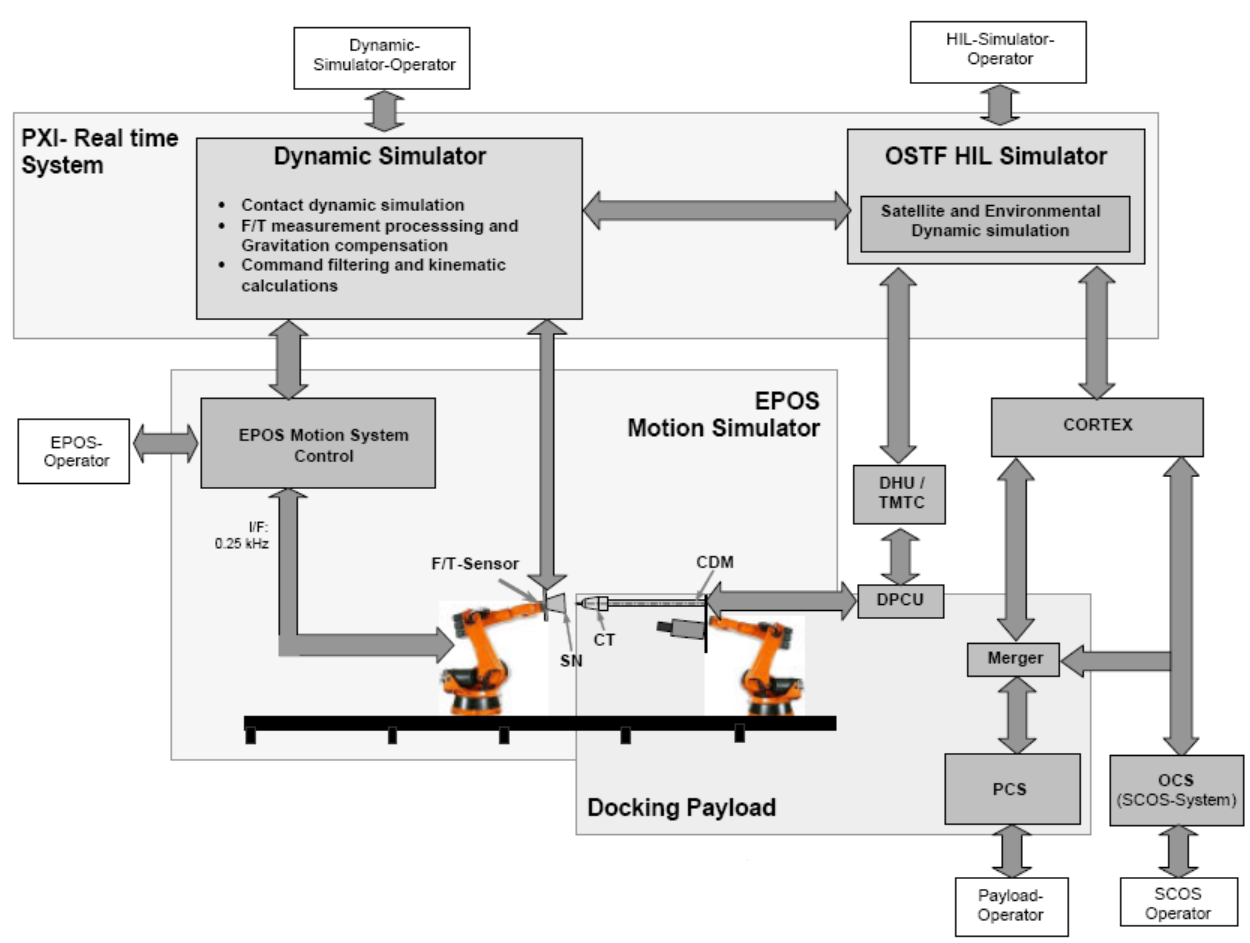

Figure 13: Possible EPOS HIL simulation scenario for OLEV

\section{E. Project Status of EPOS and Future Planning}

After dismantling the former EPOS facility in 2008 the design of the new facility was started. The new robotic hardware was installed in January 2009 and the entire facility monitoring and control system was finalized in October 2009. Since the new EPOS facility is located within GSOC's control center infrastructure it is perfectly suited for the integrated system test described above. There is a close connection to the Mission Control System (MCS) and the Payload Control System (PCS). Additionally, the test facility can be connected to the realistic communication architecture simulating delay time and jitter and the possibility to switch between teleoperation and multimission line (compare Figure 5).

The requirements for verifying camera based sensors and systems will be met after implementation of a realistic sun simulator. The requirement to verify the capture process is achieved after an Online Measurement system and contact dynamics are incorporated. Both upgrade activities had been started directly after the basic EPOS facility was installed.

\section{Conclusion}

It has been shown that operating OOS missions is technically feasible. There are solutions for all major challenges on the ground segment like teleoperation and approach navigation: A concept has been developed to reduce the delay time of the signal to $<500 \mathrm{msec}$ for LEO and $<1 \mathrm{sec}$ for GEO missions (round trip including image processing) by a direct link between the CORTEX of the antenna and the payload control system. There are also solutions to guarantee the continuity of the data link during the critical RvD maneuver using either a chain of ground stations for low earth orbit missions or a distributed network of ground stations during R-Bar approach in geostationary orbit. Furthermore, we described a navigation method based on angles-only measurements in combination with calibrated maneuvers to bridge the gap between the hand over from absolute navigation (ranging and/or GPS) to purely geometric relative navigation (stereo camera or image resolution). Finally, we described the requirements for a realistic hardware in the loop test and the specification of the new EPOS facility built at DLR as well as an integrated test set up for the missions DEOS and OLEV. Hence, DLR/GSOC and its partners are prepared to launch and operate OOS missions within the next few years. 


\section{Acknowledgments}

The authors want to thank Mr. Landzettel from the DLR Institute of Robotic and Mechatronic for general cooperation on OOS and the milestones set in the past 15 years. Many thanks also to Mr. Fehse for consultancy on the topic of RvD.

Regarding DEOS we want to thank the German Space Agency, especially the project manager Mr. Reintsema, and Mr. Naumann from SpaceTech GmbH (STI). The DEOS mission phase-A study has been awarded by the Space Agency of the Aerospace Center (DLR) and funded by the German Federal Ministry for Economics and Technology (Förderkennzeichen 50 RA 0802). With respect to OLEV many thanks to the industrial team from Kayser-Threde (Mr. Kaiser), SENER (Mr. Del Cura) and SSC (Mr. Kugelberg). Finally, we want to thank the Robo-Technology $\mathrm{GmbH}$ (Mr. Bosse) for supporting us building the new EPOS facility.

Thanks also to Mr. Maslin for carefully reading this manuscript.

\section{References}

${ }^{1}$ Settelmeyer, E., Lehrl, E., Oesterlin, W., Hartmann, R., Landzettel, K., “The Experimental Servicing Satellite - ESS”, IARP 98-c-14, 1998

${ }^{2}$ Landzettel, K., Albu-Schäffer, A., Brunner, B., Beyer, A., Gruber, R., Krämer, E., Preusche, C., Reintsema, D., Schott, J., Steinmetz, H.-J., Hirzinger, G., "ROKVISS: Verification of Advanced Light Weight Robotic Joints and Tele-Presence Concepts for Future Space Missions", ASTRA 2006 - the 9th ESA Workshop on Advanced Space Technologies for Robotics and Automation, ESA's research and technology centre, ESTEC, Noordwijk, Netherlands, 28-30 November 2006

${ }^{3}$ Landzettel, K., Preusche, C., Albu-Schäffer, A., Reintsema, D., Rebele, B., Hirzinger, G., "Robotic On-Orbit Servicing DLR's Experience and Perspective", Proceedings of the 2006 IEEE/RSJ, International Conference on Intelligent Robots and Systems, October 9 - 15, 2006, Beijing, China

${ }^{4}$ Persson, S., Bodin, P., Gill, E., Harr, J., Jörgensen, J., „PRISMA - an Autonomous Formation Flying mission”, ESA Small Satellite Systems and Services Symposium (4S), 25-29 September 2006, Sardinia, Italy

${ }^{5}$ Mulder, T.A., "Orbital Express Autonomous Rendezvous and Capture flight operations", Part 1 of 2 and Part 2 of 2 , AIAA/AAS Astrodynamics Specialist Conference and Exhibit, 18 - 21 August 2008, Honolulu, Hawaii

${ }^{6}$ Eberle, S., Ohndorf, A., Faller, R., „On-Orbit Servicing Mission Operations at GSOC”, SpaceOps 2010 Conference, Huntsville, Alabama, USA, AIAA 2010-1975 (submitted)

${ }^{7}$ Fehse, W.: Automated Rendezvous and Docking of Spacecraft, Cambridge Aerospace Series 16, Cambridge University Press, 2003

${ }^{8}$ Aida, S., Patzelt, T., Leushacke, L., Kirschner, M., Kiehling, R., "Monitoring and Mitigation of Close Proximities in Low Earth Orbit", $21^{\text {st }}$ International Symposium on Space Flight Dynamics, Toulouse, France, 2009

${ }^{9}$ Woffinden, D.C., and Geller, D.K., "Relative Angles-Only Navigation and Pose Estimation For Autonomous Orbital Rendezvous", Journal of Guidance, Control and Dynamics, Vol. 30, No. 5, 2007, pp. 1455

${ }^{10}$ Woffinden, D.C., and Geller, D.K., "Observability Criteria for Angles-Only Navigation", IEEE Transactions on Aerospace and Electronic Systems, Vol. 45, No. 3, 2009

${ }^{11}$ ESA MIV study, "Simulation Tool For Manoeuvring of a Servicing/Inspection Vehicle", ESA contract no. 11453/95/NL/JG(SC), Executive Summary, 1998

${ }^{12}$ Fehse,W., Rendezvous and Docking Workshop, Oberpfaffenhofen, Germany, 18-19 March, 2009

${ }^{13}$ Miravet, C., Pascual, L., Krouch, E., Del Cura, J.M., "An Image-based sensor system for autonomous Rendezvous with uncooperative satellites", 7th International ESA Conference on Guidance, Navigation \& Control Systems, 2-5 June, 2008

${ }^{14}$ Stastny, N.B., Bettinger, R.A., Chavez, F.R., "Comparison of the Extended and Unscented Kalman Filters for Angles Based Relative Navigation", AIAA/AAS Astrodynamics Specialist Conference and Exhibit, Honolulu, Hawaii, 18-21 August, 2008

${ }^{15}$ Schmidt, J., and Lovell, T.A., "Estimating Geometric Aspects of Relative Satellite Motion Using Angles-Only Measurements", AIAA/AAS Astrodynamics Specialist Conference and Exhibit, Honolulu, Hawaii, 18-21 August, 2008,

${ }^{16}$ D'Amico, S., and Montenbruck, O., "Proximity Operations of Formation-Flying Spacecraft Using an Eccentricity/Inclination Vector Separation”, Journal of Guidance, Control and Dynamics, Vol.29, No. 3, May-June, 2006, pp. 554-563

${ }^{17}$ Boge, T., et al.: "Hardware in the Loop Simulator von Rendezvous und Docking Manövern”, German Aerospace Congress of DGLR, Aachen, Germany, 8-10 September, 2009

${ }^{18}$ Rupp, T., Boge, T., Kiehling, R., Sellmaier, F.: "Flight Dynamics Challenges of the German On-Orbit Servicing Mission DEOS”, 21st International Symposium on Space Flight Dynamics, Toulouse, France, September/October, 2009 